\title{
Risk Aversion and Portfolio Selection in a Continuous-Time Model
}

\author{
Jianming Xia \\ Academy of Mathematics and Systems Science \\ Chinese Academy of Sciences, Beijing 100190, P.R. China \\ E-mail address: xia@amss.ac.cn
}

\begin{abstract}
The comparative statics of the optimal portfolios across individuals is carried out for a continuous-time complete market model, where the risky assets price process follows a joint geometric Brownian motion with time-dependent and deterministic coefficients. It turns out that the indirect utility functions inherit the order of risk aversion (in the Arrow-Pratt sense) from the von Neumann-Morgenstern utility functions, and therefore, a more risk-averse agent would invest less wealth (in absolute value) in the risky assets.

JEL classification: G11, D9.
\end{abstract}

Keywords: Risk aversion, Portfolio selection, Continuous-time models, Complete markets

\section{Introduction}

Portfolio selection problem is one of the classical problems in the economics of uncertainty. The optimal portfolios depend on agents' characters (preference and wealth level) and on the market's structure (the risk-free return, the return and risk of the risky assets). Various agents would have different allocations of wealth between the risk-free asset and the risky assets, due to the differences in preference and/or the differences in wealth level. The comparative statics of the optimal portfolios with respect to preference and/or wealth level has first been carried out by Arrow [1] and Pratt [13], for a static model with a risk-free asset and a risky asset. For this model, if the excess return of the risky asset is positive, then (1) the more risk-averse an agent is, the less wealth is invested in the risky asset; and (2) if an agent displays decreasing absolute (relative) risk aversion, then the amount (proportion) of wealth invested in the risky asset is increasing in wealth. 
Since then, decades have passed, but few works have been reported for dynamic models 1 as far as we know, until Borell [2]. For a continuous-time complete market model, where the risky assets price process follows a joint geometric Brownian motion, and for an agent who only consumes at the terminal time, Borell [2] has analyzed the changes of the optimal portfolios across the wealth levels. The similar conclusions that hold for the static models have been obtained there by showing the indirect utility function inherits the decreasing absolute (relative) risk aversion from the von Neumann-Morgenstern utility function.2

The purpose of this paper is to investigate how the agents' preference impacts the optimal portfolios, for the same market model as in Borell [2], but with timedependent and deterministic coefficients. As a result (see Theorem 5.1), we find that the indirect utility functions inherit the order of risk aversion from the von Neumann-Morgenstern utility functions. Observing that the vector of optimal portfolio proportions is given by the vector of log-optimal portfolio proportions multiplied by the indirect relative risk tolerance, we know it is enough for any agent to replace investments in all assets with investments in the risk-free asset and a single "mutual fund", whose portfolio is log-optimal. Based on these facts, a continuoustime version of comparative statics across individuals can be established: the more risk-averse an agent is, the less wealth is invested in the log-optimal portfolio, and hence, the less wealth in absolute value is invested in the risky assets. Using the result here, all conclusions in Borell [2] on comparisons across wealth levels can be easily recovered, as special cases.

The remainder of this paper is organized as follows: Section 2 describes the market model. Section 3 reviews the optimal solutions of portfolio selection problems, both of the dynamic programming method and the martingale/duality approach are included. In particular, it is pointed out that the amount of wealth invested in the log-optimal portfolio equals the indirect absolute risk tolerance. Section 4 gives some representations of the indirect absolute risk tolerance and derives a nonlinear parabolic PDE (partial differential equation) for the indirect absolute risk tolerance

\footnotetext{
${ }^{1}$ Except for some specific cases such as constant absolute (relative) risk aversion, in which the solutions can be explicitly worked out.

${ }^{2}$ The indirect utility function also inherits the increasing relative risk aversion from the von Neumann-Morgenstern utility function. The preservation of decreasing (increasing) absolute risk aversion has been presented by Gollier [5], for static and complete models.
} 
function. Section 5 presents the main result of this paper and Section 6 recovers the conclusions of Borell [2]. The other sections provide the technical arguments.

We shall make use of the following notation: $M^{\top}$ stands for transposition of a

vector or a matrix $M ;|\zeta|=\sqrt{\zeta^{\top} \zeta}$ is the usual Euclidean norm for a vector $\zeta ; \mathbb{1}$ is the $n$-dimensional vector with each component equals 1 ; and for a domain $\mathcal{D} \subset$ $[0, T] \times(0, \infty), C^{1, \infty}(\mathcal{D})$ denotes the set of all functions $f: \mathcal{D} \rightarrow \mathbb{R}$ such that $f(t, x)$ are continuously differentiable with respect to $t$ and infinite times differentiable with respect to $x$, for all $(t, x) \in \mathcal{D} ; C(\mathcal{D})$ denotes the set of all continuous functions $f: \mathcal{D} \rightarrow \mathbb{R}$.

\section{The Financial Market}

We consider the typical setup for a continuous-time financial market economy on the finite time span $[0, T]$. The financial market consists of a risk-free asset and $n$ risky assets. The risk-free asset's price process $S^{0}(t)$ evolves according to the following equation:

$$
d S^{0}(t)=S^{0}(t) r(t) d t, \quad S^{0}(0)=1,
$$

where $r(t)$ is the interest rate process. The $i$-th risky asset's price process $S^{i}(t)$ satisfies the following equation:

$$
d S^{i}(t)=S^{i}(t)\left(b^{i}(t) d t+\sum_{j=1}^{n} \sigma^{i j}(t) d B^{j}(t)\right), \quad S^{i}(0)>0,1 \leq i \leq n .
$$

Here $B(t)=\left(B^{1}(t), \cdots, B^{n}(t)\right)^{\top}$ is an $n$-dimensional standard Brownian motion defined on a probability space $\left(\Omega, \mathcal{F},\left(\mathcal{F}_{t}\right), \mathbb{P}\right)$. The information structure $\left(\mathcal{F}_{t}\right)$ is the $\mathbb{P}$-augmentation of the filtration generated by $B(t)$ and $\mathcal{F}=\mathcal{F}_{T}$. Set $b(t)=$ $\left(b^{1}(t), \cdots, b^{n}(t)\right)^{\top}$ and $\sigma(t)=\left(\sigma^{i j}(t)\right)_{1 \leq i, j \leq n}$. In this paper, we always assume the coefficients $r(t), b(t)$, and $\sigma(t)$ satisfy the following condition:

Assumption 2.1 (1) All of $r(t), b^{i}(t)(1 \leq i \leq n)$, and $\sigma^{i j}(t)(1 \leq i, j \leq n)$ are deterministic and continuous functions of $t$, on $[0, T]$; (2) the matrix $\sigma(t)$ is nonsingular for each $t$ and there exists a constant $c>0$ such that $\zeta^{\top} \sigma(t)^{-1} \zeta \geq c|\zeta|^{2}$ for all $t \in[0, T]$ and $\zeta \in \mathbb{R}^{n}$.

In the above setting, the financial market is complete and admits a unique equivalent martingale measure, or risk-neutral measure, denoted by $\mathbb{Q}$, whose density 
process is $\left.\frac{d \mathbb{Q}}{d \mathbb{P}}\right|_{\mathcal{F}_{t}}=\rho(t)$, where

$$
\begin{aligned}
& \rho(t):=\exp \left\{-\int_{0}^{t} \theta(\tau)^{\top} d B(\tau)-\int_{0}^{t} \frac{|\theta(\tau)|^{2}}{2} d \tau\right\}, \\
& \theta(t):=\sigma(t)^{-1}(b(t)-r(t) \mathbb{1}) .
\end{aligned}
$$

By the Girsanov's Theorem,

$$
B^{*}(t):=B(t)+\int_{0}^{t} \theta(\tau)^{\top} d \tau
$$

is an $n$-dimensional standard Brownian motion under $\mathbb{Q}$. Obviously, each risky asset price process satisfies the following equation:

$$
d S^{i}(t)=S^{i}(t)\left(r(t) d t+\sum_{j=1}^{n} \sigma^{i j}(t) d B^{* j}(t)\right), \quad 1 \leq i \leq n .
$$

The state-price deflator $H$ is defined by

$$
H(t)=\exp \left\{-\int_{0}^{t} r(\tau) d \tau\right\} \rho(t) .
$$

It is well known that $S^{i}(t) H(t)$ is a martingale, for $i=1, \ldots, n$.

\section{Utility Maximization}

In this paper, a von Neumann-Morgenstern utility function $U:(0, \infty) \rightarrow \mathbb{R}$ is supposed to be a strictly increasing, strictly concave, and twice-continuously-differentiable function that satisfies the Inada condition

$$
U^{\prime}(0)=\lim _{x \downarrow 0} U^{\prime}(x)=\infty \quad \text { and } \quad U^{\prime}(\infty)=\lim _{x \uparrow \infty} U^{\prime}(x)=0 .
$$

Given a von Neumann-Morgenstern utility function $U$, the Arrow-Pratt coefficient of absolute risk aversion at $x$ is $-\frac{U^{\prime \prime}(x)}{U^{\prime}(x)}$, and the absolute risk tolerance is $-\frac{U^{\prime}(x)}{U^{\prime \prime}(x)}$. Accordingly, $-\frac{x U^{\prime \prime}(x)}{U^{\prime}(x)}$ is the Arrow-Pratt coefficient of relative risk aversion and $-\frac{U^{\prime}(x)}{x U^{\prime \prime}(x)}$ is the relative risk tolerance. Let us introduce an assumption on $U$ that will be used.

Assumption 3.1 The absolute risk tolerance function of $U$ satisfies the linear growth condition, that is, there is a constant $c>0$ such that

$$
-\frac{U^{\prime}(x)}{U^{\prime \prime}(x)} \leq c(1+x), \text { for all } x>0 .
$$


Following Merton [11], we assume that (1) there are no transaction costs, taxes, or asset indivisibility; (2) the agents are price takers; (3) short sales of all assets, with full use of proceeds, are allowed; and (4) trading in assets takes place continuously in time.

We consider an agent who consumes only at the terminal time and whose utility function for the consumption at the terminal time is $U$. At any given starting time $t$, the preference of the agent for the terminal consumption can be represented by the expected utility $\mathbb{E}_{t}[U(X(T))]$, where $X(T)$ is the value of the terminal wealth and $\mathbb{E}_{t}$ is the conditional expectation operator at time $t$. The agent is allowed to allocate the wealth between the risk-free asset and the risky assets so as to maximize the expected utility. That is, the agent solves the dynamic investment problem

$$
\max _{(\phi(s))} \mathbb{E}_{t}[U(X(T))]
$$

subject to

$$
\left\{\begin{array}{l}
d X(s)=\left[X(s) r(s)+\phi(s)^{\top}(b(s)-r(s) \mathbb{1})\right] d s+\phi(s)^{\top} \sigma(s) d B(s), \\
X(s) \geq 0, \quad s \in[t, T] \\
X(t)=x,
\end{array}\right.
$$

where $\phi(s)=\left(\phi^{1}(s), \cdots, \phi^{n}(s)\right)^{\top}$ is the vector of values of wealth invested in the risky assets at time $s \in[t, T], x>0$ is the value of wealth at the starting time $t$. The first constraint in (3.2) is the dynamic budget constraint determining the evolution of the wealth process. The second constraint in (3.2) is the nonnegative wealth constraint ruling out the possibility of create something out of nothing.

Following Cox and Huang [3], see also Karatzas et al [7] and Pliska [12], we can transform the dynamic problem (3.1) into a static one:

$$
\begin{aligned}
\max _{X(T) \geq 0} & \mathbb{E}_{t}[U(X(T))] \\
\text { subject to } & \mathbb{E}_{t}\left[\frac{H(T)}{H(t)} X(T)\right] \leq x .
\end{aligned}
$$

In other words, the dynamic budget constraint in (3.2) can be replaced by a static budget constraint. 
Since $U^{\prime}(0)=\infty$, the solution $X(T)$ of problem (3.3) is strictly positive and the first-order condition is

$$
U^{\prime}(X(T))=\lambda(t, x) \frac{H(T)}{H(t)},
$$

where the Lagrangian multiplier $\lambda(t, x)>0$. Henceforth, we use $I$ to denote the inverse marginal utility function $U^{\prime-1}$, that is, $U^{\prime}(I(y))=y$, for all $y>0$. Obviously, $I$ is strictly decreasing and continuously differentiable on $(0, \infty)$, and

$$
I(0)=\lim _{y \downarrow 0} I(y)=\infty, \quad I(\infty)=\lim _{y \uparrow \infty} I(y)=0 .
$$

With this notation, the solution $X(T)$ of problem (3.3) is

$$
X(T)=I\left(\lambda(t, x) \frac{H(T)}{H(t)}\right) .
$$

Furthermore, since $U$ is increasing, the budget constraint is binding:

$$
\mathbb{E}_{t}\left[\frac{H(T)}{H(t)} X(T)\right]=x,
$$

that is, the Lagrangian multiplier $\lambda(t, x)$ satisfies the following equation:

$$
\mathbb{E}_{t}\left[\frac{H(T)}{H(t)} I\left(\lambda(t, x) \frac{H(T)}{H(t)}\right)\right]=x .
$$

For any $t \in[0, T]$ and $y>0$, set

$$
\mu(t, y)=\mathbb{E}_{t}\left[\frac{H(T)}{H(t)} I\left(y \frac{H(T)}{H(t)}\right)\right] .
$$

Obviously, the independent increments of Brownian motion yield that $\mu$ is a deterministic function defined on $[0, T] \times(0, \infty)$, and for any given $t, \mu(t, y)$ is strictly decreasing with respect to $y$. Particularly, $\mu(T, y)=I(y)$, for $y>0$. We can see from (3.6) and the definition of $\mu(t, y)$ that, for all $t \in[0, T]$ and $x>0$,

$$
\mu(t, \lambda(t, x))=x .
$$

Hence, $\lambda(t, x)$ is also a deterministic function defined on $[0, T] \times(0, \infty)$, and for any given $t, \lambda(t, x)$ is strictly decreasing with respect to $x$. Particularly, $\lambda(T, x)=U^{\prime}(x)$, for $x>0$.

Let $u(t, x)$ denote the value function of the problem (3.1), or the indirect utility function, given that the value of wealth at the starting time $t$ is $x$, that is

$$
u(t, x)=\mathbb{E}_{t}\left[U\left(I\left(\lambda(t, x) \frac{H(T)}{H(t)}\right)\right)\right],
$$


for all $(t, x) \in[0, T] \times(0, \infty)$. The independent increments of Brownian motion yield that $u$ is a deterministic function defined on $[0, T] \times(0, \infty)$. For the indirect utility function, we have the following proposition:

Proposition 3.1 Under Assumptions 2.1 and 3.1,

$$
u \in C^{1, \infty}([0, T) \times(0, \infty)) \cap C([0, T] \times(0, \infty)) .
$$

Proof. See Appendix A,

Under the conditions of the preceding proposition, following Merton [11], the principle of optimality leads to the following HJB (Hamilton-Jacobi-Bellman) equation for $u$ :

$$
\max _{\phi}\left\{u_{t}+\left[r x+\phi^{\top}(b-r \mathbb{1})\right] u_{x}+\frac{1}{2} \phi^{\top} \sigma \sigma^{\top} \phi u_{x x}\right\}=0,
$$

on $[0, T) \times(0, \infty)$, with terminal condition

$$
u(T, x)=U(x), \quad \text { for all } x>0
$$

It is well known that $u_{x}(t, x)>0$ and $u_{x x}(t, x)<0$, for all $(t, x) \in[0, T] \times(0, \infty)$. The first-order condition for the maximality in (3.10) implies that the optimal portfolio policy $\hat{\phi}$ is in the following feedback form:

$$
\hat{\phi}(t, x)=-\frac{u_{x}(t, x)}{u_{x x}(t, x)}\left(\sigma(t) \sigma(t)^{\top}\right)^{-1}(b(t)-r(t) \mathbb{1}),
$$

that is,

$$
\hat{\phi}(t, x)=f(t, x)\left(\sigma(t) \sigma(t)^{\top}\right)^{-1}(b(t)-r(t) \mathbb{1}),
$$

where

$$
f(t, x)=-\frac{u_{x}(t, x)}{u_{x x}(t, x)}, \quad \text { for all }(t, x) \in[0, T] \times(0, \infty) .
$$

Obviously, $f(t, x)>0$, for all $(t, x) \in[0, T] \times(0, \infty) . f(t, x)$ is the absolute risk tolerance of the indirect utility function. In this paper, we call it the indirect absolute risk tolerance function. Accordingly, we call $\frac{f(t, x)}{x}$ the indirect relative risk tolerance function.

In view of (3.12),

$$
\frac{\hat{\phi}(t, x)}{x}=\frac{f(t, x)}{x}\left(\sigma(t) \sigma(t)^{\top}\right)^{-1}(b(t)-r(t) \mathbb{1}),
$$


for all $(t, x) \in[0, T) \times(0, \infty)$. $\frac{\hat{\phi}(t, x)}{x}$ is the vector of optimal portfolio proportions, whose components represent the proportions of total wealth held in the risky assets. In particular, for logarithmic utility function $U(x)=\log x$, whose relative risk

tolerance is constant and equals 1 , it is well know 3 that the indirect relative risk tolerance $\frac{f(t, x)}{x}=1$, for all $(t, x) \in[0, T] \times(0, \infty)$, and hence, by (3.13), the vector of optimal portfolio proportions is $\left(\sigma(t) \sigma(t)^{\top}\right)^{-1}(b(t)-r(t) \mathbb{1})$, which, hereafter, is called the vector of log-optimal portfolio proportions.

Notice that, for any von Neumann-Morgenstern utility function $U$, the vector of optimal portfolio proportions is given by the vector of log-optimal portfolio proportions multiplied by the indirect relative risk tolerance. This means effectively that it is enough for any agent to replace investments in all assets with investments in the risk-free asset and a single "mutual fund", whose portfolio is log-optimal. Different agents would have different weights between the log-optimal portfolio and the risk-free asset, depending on their indirect relative risk tolerance. The weight of total wealth invested in the log-optimal portfolio equals the indirect relative risk tolerance. The larger the indirect relative risk tolerance is, the larger weight is invested in the log-optimal portfolio. Accordingly, the amount of wealth invested in the log-optimal portfolio equals the indirect absolute risk tolerance. The larger the indirect absolute risk tolerance is, the more wealth is invested in the log-optimal portfolio.

\section{Indirect Absolute Risk Tolerance Functions}

Now we investigate the indirect absolute risk tolerance function $f$.

In this section, we let $\hat{X}(T, x)$ denote the solution of problem (3.3). Note the solution depends on both of the starting wealth $x$ and the starting time $t$. For simplicity, however, we suppress the explicit dependence on $t$ in the notation.

The Lagrangian multiplier $\lambda(t, x)$ gives the marginal, or shadow, value of relaxing the static budget constraint in (3.3). It therefore equals the agent's marginal utility of wealth at the optimum, that is

$$
\lambda(t, x)=u_{x}(t, x)
$$

\footnotetext{
${ }^{3}$ See Merton [10].
} 
In view of (3.4), the first-order condition is

$$
U^{\prime}(\hat{X}(T, x))=u_{x}(t, x) \frac{H(T)}{H(t)} .
$$

Moreover, the budget constraint is binding:

$$
\mathbb{E}_{t}\left[\hat{X}(T, x) \frac{H(T)}{H(t)}\right]=x .
$$

Lemma 4.1 Under Assumptions 2.1 and 3.1, we have for any $t \in[0, T)$ that

$$
\mathbb{E}_{t}\left[\frac{\partial \hat{X}(T, x)}{\partial x} \frac{H(T)}{H(t)}\right]=1, \text { for all } x>0
$$

Proof. We can obtain (4.3) by differentiating formally the both sides of (4.2) with respect to $x$. For a rigorous proof, see Appendix B.

Differentiating (4.1) with respect to $x$ yields

$$
U^{\prime \prime}(\hat{X}(T, x)) \frac{\partial \hat{X}(T, x)}{\partial x}=u_{x x}(t, x) \frac{H(T)}{H(t)},
$$

and consequently, by (4.1) again,

$$
-\frac{U^{\prime}(\hat{X}(T, x))}{U^{\prime \prime}(\hat{X}(T, x))}=-\frac{u_{x}(t, x)}{u_{x x}(t, x)} \frac{\partial \hat{X}(T, x)}{\partial x}=f(t, x) \frac{\partial \hat{X}(T, x)}{\partial x} .
$$

Proposition 4.1 Under Assumptions [2.1 and [3.1, for all $t \in[0, T]$ and $x>0$,

$$
\begin{aligned}
f(t, x) & =\mathbb{E}_{t}\left[-\frac{U^{\prime}(\hat{X}(T, x))}{U^{\prime \prime}(\hat{X}(T, x))} \frac{H(T)}{H(t)}\right] \\
& =\mathbb{E}_{t}\left[-\frac{U^{\prime}\left(I\left(\lambda(t, x) \frac{H(T)}{H(t)}\right)\right)}{U^{\prime \prime}\left(I\left(\lambda(t, x) \frac{H(T)}{H(t)}\right)\right)} \frac{H(T)}{H(t)}\right] .
\end{aligned}
$$

Proof. We can obtain (4.5) from (4.4) and Lemma 4.1. From (3.5), we get (4.6) as well.

Remark 4.1 Gollier [5] obtained the same conclusion as in the preceding proposition, for static models.

Apart from the representations (4.5)-(4.6), the indirect absolute risk tolerance function $f$ satisfies a nonlinear parabolic PDE and is continuous on the boundary, as showed in the next proposition. 
Proposition 4.2 Under Assumptions 2.1 and 3.1,

$$
f \in C^{1, \infty}([0, T) \times(0, \infty)) \cap C([0, T] \times(0, \infty)),
$$

and satisfies $P D E$

$$
\frac{1}{2}|\theta|^{2} f^{2} f_{x x}+r x f_{x}+f_{t}-r f=0,
$$

on $[0, T) \times(0, \infty)$, with terminal condition

$$
f(T, x)=-\frac{U^{\prime}(x)}{U^{\prime \prime}(x)}, \quad \text { for all } x>0 .
$$

Proof. See Appendix B,

In the following part of this section, the optimal wealth process of problem (3.1) is denoted by $\{\hat{X}(s), s \in[t, T]\}$. It was first pointed out by Cox and Leland [4] that $\{f(s, \hat{X}(s)) H(s), s \in[t, T]\}$ is a local martingale when the risky asset price process is a geometric Brownian motion. He and Huang [6] observed it is a general property of an optimal consumption-portfolio policy (for case of a static model, refer to Gollier [5]). In what follows, we are going to show $\{f(s, \hat{X}(s)) H(s), s \in[t, T]\}$ is a martingale.

Proposition 4.3 Under Assumptions 2.1 and 3.1, $\{f(s, \hat{X}(s)) H(s), s \in[t, T]\}$ is a martingale, for each $t \in[0, T)$ and $x>0$.

Proof. By Lemma B.3, $\{f(s, \hat{X}(s)) H(s), s \in[t, T]\}$ is a nonnegative local martingale, and hence a supermartingale. So it suffices to show

$$
\mathbb{E}_{t}[f(T, \hat{X}(T)) H(T)]=f(t, x) H(t) .
$$

This can be easily obtained from (4.5)).

\section{Comparisons Across Individuals}

Apart from an agent with utility function $U$ as described in the previous sections, we consider another agent whose utility function is $V$. Just like the arguments in the previous sections, we will use the following assumption: 
Assumption 5.1 The absolute risk tolerance function of $V$ satisfies the linear growth condition, that is, there is a constant $c>0$ such that

$$
-\frac{V^{\prime}(x)}{V^{\prime \prime}(x)} \leq c(1+x), \text { for all } x>0
$$

The agent whose utility function is $V$ solves the following dynamic investment problem:

$$
\max _{(\varphi(s))} \mathbb{E}_{t}[V(X(T))]
$$

subject to

$$
\left\{\begin{array}{l}
d X(s)=\left[X(s) r(s)+\varphi^{\top}(s)(b(s)-r(s) \mathbb{1})\right] d s+\varphi^{\top}(s) \sigma(s) d B(s) \\
X(s) \geq 0, \quad \text { for } s \in[t, T] \\
X_{t}=x
\end{array}\right.
$$

where $\varphi(s)=\left(\varphi^{1}(s), \cdots, \varphi^{n}(s)\right)^{\top}$ is the vector of values of wealth invested in the risky assets at time $s \in[t, T]$.

Let $v(t, x)$ denote the indirect utility function for problem (5.1). From Proposition 3.1, we know $v \in C^{1, \infty}([0, T) \times(0, \infty)) \cap C([0, T] \times(0, \infty))$, provided Assumptions 2.1 and 5.1 are satisfied. The corresponding indirect absolute risk tolerance function is $g(t, x)=-\frac{v_{x}(t, x)}{v_{x x}(t, x)}$, and the optimal portfolio policy $\hat{\varphi}$ is in the following feedback form:

$$
\hat{\varphi}(t, x)=g(t, x)\left(\sigma(t) \sigma(t)^{\top}\right)^{-1}(b(t)-r(t) \mathbb{1}) .
$$

Given two utility functions $U$ and $V$, we say $U$ is more risk-averse than $V$, or $U$ is less risk-tolerant than $V$, if $-\frac{U^{\prime \prime}(x)}{U^{\prime}(x)} \geq-\frac{V^{\prime \prime}(x)}{V^{\prime}(x)}$, that is, $-\frac{U^{\prime}(x)}{U^{\prime \prime}(x)} \leq-\frac{V^{\prime}(x)}{V^{\prime \prime}(x)}$, for every $x>0$. It is well known that $U$ is more risk-averse than $V$ if and only if there exists an increasing concave function $F$ such that $U(x)=F(V(x))$, for all $x$; that is, $U$ is a concave transformation of $V$ (In other words, $U$ is "more concave" than $V$.)

Remark 5.1 Obviously, if $V$ satisfies Assumption 5.1 and $U$ is more risk-averse that $V$, then $U$ satisfies Assumption 3.1.

Now we are ready to report the main result of this paper. 
Theorem 5.1 Under Assumptions 2.1 and 5.1, assume further that $U$ is more riskaverse than $V$, then $f(t, x) \leq g(t, x)$, for all $t \in[0, T)$ and $x>0$.

Proof. See Section 8 ,

The preceding theorem shows: If the agent with utility function $U(x)$ is more risk-averse than the agent with utility function $V(x)$, then, for each time $t \in[0, T)$, the corresponding indirect utility function $u(t, x)$ is more risk-averse than $v(t, x)$ as well. According to the discussion at the end of Section 3, at each time $t \in[0, T)$, if the agents have the same value of wealth, then the former agent invests less in the log-optimal portfolio (and hence, less in absolute value of wealth in the risky assets) than the later. So, we establish a dynamic, continuous-time version of the comparative statics of the optimal portfolios across individuals.

Under the conditions of the preceding theorem, by Remark 5.1 and Proposition 4.2, $f$ and $g$ satisfy the same PDE on $[0, T) \times(0, \infty)$. Moreover, at the terminal time,

$$
f(T, x)=-\frac{U^{\prime}(x)}{U^{\prime \prime}(x)} \leq-\frac{V^{\prime}(x)}{V^{\prime \prime}(x)}=g(T, x), \quad \text { for all } x>0 .
$$

It seems that we can apply the techniques of maximum principles for parabolic PDEs to prove the assertion of the previous theorem. However, due to the nonlinearity of PDE (4.7) and unboundedness of the domain, the techniques of maximum principles can not be directly used to $f$ and $g$. In order to overcome this point, we approximate $f$ with a sequence $\left\{f^{(m)}, m \geq 2\right\}$, which satisfy the PDEs with bounded domains. For these PDEs with bounded domains, we can use the techniques of maximum principles, then by approximation, the the preceding theorem can be proved. The approximating sequence is constructed in Section 7 , and the proof of the preceding theorem is completed in Section 8 ,

\section{Comparisons Across Wealth Levels}

In this section, we recover all conclusions in Borell [2], based on Proposition 4.1 and Theorem 5.1 .

A utility function $U$ is called to exhibit decreasing absolute risk aversion (henceforth, DARA) [resp. increasing absolute risk aversion (henceforth, IARA)], if $-\frac{U^{\prime \prime}(x)}{U^{\prime}(x)}$ 
is decreasing [resp. increasing] with respect to $x$. Accordingly, $U$ is called to exhibit decreasing relative risk aversion (henceforth, DRRA) [resp. increasing relative risk aversion (henceforth, IRRA)] if $-\frac{x U^{\prime \prime}(x)}{U^{\prime}(x)}$ is decreasing [resp. increasing] with respect to $x$.

Theorem 6.1 Under Assumptions 2.1 and 3.1, if $U$ exhibits DARA, then for each $t, u(t, \cdot)$ exhibits DARA, namely, $f(t, x)$ is increasing with respect to $x$.

Proof. We have known that $I$ is strictly decreasing, and for each $t, \lambda(t, x)$ is strictly decreasing with respect to $x$. Then the assertions follows from (4.6).

Remark 6.1 The preservation of DARA has already been reported by Borell [2], for a continuous-time complete model; see Gollier [5, pp.209-210], for a static complete model. The method used here is same to that of Gollier [5].4

We can see from the preceding theorem and the discussion at the end of Section 3 that, if the utility function of the agent exhibits DARA, then the amount of wealth invested in the log-optimal portfolio is increasing as the total wealth rises.

As for the relative risk aversion, we have the following theorem, whose conclusion has already been reported by Borell [2]. The methodology here, however, is different from there. According to the method of Borell [2], the IRRA case is much more complicate than the DRRA case. According to the method here, however, both cases can be easily dealt with, based on Theorem 5.1 .

Theorem 6.2 Under Assumptions 2.1 and 3.1, we have the following assertions:

(a) If $U$ exhibits DRRA, then for each $t, u(t, \cdot)$ exhibits DRRA, namely, $\frac{f(t, x)}{x}$ is increasing with respect to $x$;

(b) If $U$ exhibits IRRA, then for each $t, u(t, \cdot)$ exhibits IRRA, namely, $\frac{f(t, x)}{x}$ is decreasing with respect to $x$.

\footnotetext{
${ }^{4}$ Gollier [5], for a static complete model, has showed the preservation of IARA. But in our settings, as observed by Borell [2, p.144], the assumption $U^{\prime}(0)=\infty$ totally eliminates utility functions $U$ exhibiting IARA (see also Lemma A.1). We believe, in our continuous-time setting, the preservation of IARA can be proved as well, by considering the utility functions defined on the whole real line $(-\infty, \infty)$, instead of the positive real line $(0, \infty)$.
} 
Proof. Suppose $U$ exhibits DRRA. For any constant $\gamma>1$, consider the utility function $V$ defined by $V(x)=U(\gamma x)$, for all $x>0$. Obviously, we have

$$
-\frac{x V^{\prime \prime}(x)}{V^{\prime}(x)}=-\frac{\gamma x U^{\prime \prime}(\gamma x)}{U^{\prime}(\gamma x)} \leq-\frac{x U^{\prime \prime}(x)}{U^{\prime}(x)},
$$

which yields $U$ is more risk averse than $V$. Then by Theorem 5.1,

$$
f(t, x) \leq g(t, x), \quad \text { for all }(t, x) \in[0, T] \times(0, \infty)
$$

Moreover, it is easy to see $v(t, x)=u(t, \gamma x)$, for all $t \in[0, T]$ and $x>0$. By computation, $g(t, x)=\frac{f(t, \gamma x)}{\gamma}$. Thus we get from (6.1) that $\frac{f(t, x)}{x} \leq \frac{f(t, \gamma x)}{\gamma x}$, for all $t \in[0, T]$ and $x>0$. By the arbitrariness of $\gamma>1$, we have proved assertion (a). Assertion (b) can be proved by letting $\gamma \in(0,1)$ and by the same way.

We can see from the preceding theorem and the discussion at the end of Section 3 that, if the utility function of the agent exhibits DRRA (resp. IRRA), then the weight of wealth invested in the log-optimal portfolio is increasing (resp. decreasing) as the total wealth rises.

\section{Approximation}

We now begin to construct the approximating sequence $\left\{f^{(m)}, m \geq 2\right\}$.

Given a utility function $U$, for each $m \geq 2$ and for each $t \in[0, T)$, we consider the following constrained problem:

$$
\max _{(\phi(s))} \mathbb{E}_{t}[U(X(T))]
$$

subject to

$$
\left\{\begin{array}{l}
d X(s)=\left[X(s) r(s)+\phi^{\top}(s)(b(s)-r(s) \mathbb{1})\right] d s+\phi^{\top}(s) \sigma(s) d B(s) \\
\frac{1}{m} \leq X(T) \leq m \\
X(s) \geq 0, \quad \text { for } s \in[t, T] \\
X_{t}=x
\end{array}\right.
$$

where $\phi(s)=\left(\phi^{1}(s), \cdots, \phi^{n}(s)\right)^{\top}$ is the vector of values of wealth invested in risky assets at time $s \in[t, T]$, and $\frac{1}{m} e^{-\int_{t}^{T} r(s) d s}<x<m e^{-\int_{t}^{T} r(s) d s}$. 
Following an identical discussion as in Section 3 , we can see the dynamic problem (7.1) can be transformed into a static one:

$$
\begin{array}{ll}
\max _{\frac{1}{m} \leq X(T) \leq m} & \mathbb{E}_{t}[U(X(T))] \\
\text { subject to } & \mathbb{E}_{t}\left[\frac{H(T)}{H(t)} X(T)\right] \leq x .
\end{array}
$$

By a similar discussion as in Section 3 , the solution $X^{(m)}(T)$ is,

$$
X^{(m)}(T)=\frac{1}{m} \vee I\left(\lambda^{(m)}(t, x) \frac{H(T)}{H(t)}\right) \wedge m
$$

where the Lagrangian multiplier $\lambda^{(m)}(t, x)>0$ and we use the following notation:

$$
\frac{1}{m} \vee x \wedge m= \begin{cases}\frac{1}{m}, & \text { for } x \leq \frac{1}{m} \\ x, & \text { for } x \in\left(\frac{1}{m}, m\right) ; \\ m, & \text { for } x \geq m .\end{cases}
$$

Moreover, the static budget constraint is binding:

$$
\mathbb{E}_{t}\left[\frac{H(T)}{H(t)} X^{(m)}(T)\right]=x
$$

that is,

$$
\mathbb{E}_{t}\left[\frac{H(T)}{H(t)}\left(\frac{1}{m} \vee I\left(\lambda^{(m)}(t, x) \frac{H(T)}{H(t)}\right) \wedge m\right)\right]=x
$$

For any $y>0$, define

$$
\mu^{(m)}(t, y)=\mathbb{E}_{t}\left[\frac{H(T)}{H(t)}\left(\frac{1}{m} \vee I\left(y \frac{H(T)}{H(t)}\right) \wedge m\right)\right] .
$$

The independent increments of Brownian motion yield $\mu^{(m)}$ is a deterministic function defined on $[0, T] \times(0, \infty)$. Obviously, for any given $t \in[0, T), \mu^{(m)}(t, y)$ is continuous and strictly decreasing with respect to $y$, on $(0, \infty)$, and

$$
\lim _{y \downarrow 0} \mu^{(m)}(t, y)=m e^{-\int_{t}^{T} r(s) d s}, \quad \lim _{y \uparrow \infty} \mu^{(m)}(t, y)=\frac{1}{m} e^{-\int_{t}^{T} r(s) d s} .
$$

By (7.4) and the definition of $\mu^{(m)}$,

$$
\mu^{(m)}\left(t, \lambda^{(m)}(t, x)\right)=x,
$$


for any $\frac{1}{m} e^{-\int_{t}^{T} r(s) d s}<x<m e^{-\int_{t}^{T} r(s) d s}$. Therefore, $\lambda^{(m)}$ is a deterministic function defined on $\mathcal{D}^{(m)} \cup \mathcal{T}^{(m)}$, where

$$
\begin{aligned}
& \mathcal{D}^{(m)}=\left\{(t, x): \frac{1}{m} e^{-\int_{t}^{T} r(s) d s}<x<m e^{-\int_{t}^{T} r(s) d s}, t \in[0, T)\right\}, \\
& \mathcal{T}^{(m)}=\left\{(T, x): \frac{1}{m}<x<m\right\} .
\end{aligned}
$$

Moreover, for any given $t \in[0, T), \lambda^{(m)}(t, x)$ is continuous and strictly decreasing with respect to $x$, on $\left(\frac{1}{m} e^{-\int_{t}^{T} r(s) d s}, m e^{-\int_{t}^{T} r(s) d s}\right)$, and

$$
\lim _{x \downarrow \frac{1}{m} e^{-\int_{t}^{T} r(s) d s}} \lambda^{(m)}(t, x)=\infty, \quad \lim _{x \uparrow m e^{-\int_{t}^{T} r(s) d s}} \lambda^{(m)}(t, x)=0 .
$$

In view of (7.3), the indirect utility function of problem (7.2) is

$$
u^{(m)}(t, x)=\mathbb{E}_{t}\left[U\left(\frac{1}{m} \vee I\left(\lambda^{(m)}(t, x) \frac{H(T)}{H(t)}\right) \wedge m\right)\right] .
$$

The independent increments of Brownian motion yield that $u^{(m)}$ is a deterministic function defined on $\mathcal{D}^{(m)} \cup \mathcal{T}^{(m)}$.

Proposition 7.1 Under Assumption 2.1, for each $m \geq 2$,

$$
u^{(m)} \in C^{1, \infty}\left(\mathcal{D}^{(m)}\right) \cap C\left(\mathcal{D}^{(m)} \cup \mathcal{T}^{(m)}\right)
$$

Proof. See Appendix C.

The preceding proposition implies $u^{(m)}$ satisfies the following HJB equation:

$$
\max _{\phi}\left\{u_{t}^{(m)}+\left[r x+\phi^{\top}(b-r \mathbb{1})\right] u_{x}^{(m)}+\frac{1}{2} \phi^{\top} \sigma \sigma^{\top} \phi u_{x x}^{(m)}\right\}=0,
$$

on $\mathcal{D}^{(m)}$, with terminal condition

$$
u^{(m)}(T, x)=U^{(m)}(x), \quad \text { for } x \in\left(\frac{1}{m}, m\right)
$$

For each $m \geq 2$, the indirect absolute risk tolerance function is

$$
f^{(m)}(t, x)=-\frac{u_{x}^{(m)}(t, x)}{u_{x x}^{(m)}(t, x)}, \quad \text { for }(t, x) \in \mathcal{D}^{(m)} \cup \mathcal{T}^{(m)} .
$$


Proposition 7.2 Under Assumptions 2.1 and 3.1, for each $m \geq 2$,

$$
f^{(m)} \in C^{1, \infty}\left(\mathcal{D}^{(m)}\right) \cap C\left(\mathcal{D}^{(m)} \cup \mathcal{T}^{(m)}\right),
$$

and satisfies $P D E$

$$
\frac{1}{2}|\theta|^{2}\left(f^{(m)}\right)^{2} f_{x x}^{(m)}+r x f_{x}^{(m)}+f_{t}^{(m)}-r f^{(m)}=0,
$$

on $\mathcal{D}^{(m)}$, with terminal condition

$$
f^{(m)}(T, x)=-\frac{U^{\prime}(x)}{U^{\prime \prime}(x)}, \quad \text { for } x \in\left(\frac{1}{m}, m\right),
$$

and boundary conditions

$$
\left\{\begin{array}{cl}
\lim _{(s, x) \rightarrow\left(t, m e^{-\int_{t}^{T} r(s) d s}\right)} f^{(m)}(s, x)=0, & \text { for } t \in[0, T) ; \\
\lim _{(s, x) \rightarrow\left(t, \frac{1}{m} e^{-\int_{t}^{T} r(s) d s}\right)} f^{(m)}(s, x)=0, & \text { for } t \in[0, T) .
\end{array}\right.
$$

Moreover, we have

$$
\begin{aligned}
& \limsup _{(s, x) \rightarrow\left(T, \frac{1}{m}\right)} f^{(m)}(s, x)=-\frac{U^{\prime}\left(\frac{1}{m}\right)}{U^{\prime \prime}\left(\frac{1}{m}\right)}, \\
& \limsup _{(s, x) \rightarrow(T, m)} f^{(m)}(s, x)=-\frac{U^{\prime}(m)}{U^{\prime \prime}(m)} .
\end{aligned}
$$

Proof. See Appendix C.

The sequence $f^{(m)}$ constructed above is indeed approximating $f$, as the following proposition shows.

Proposition 7.3 Under Assumptions 2.1 and 3.1, $\lim _{m \rightarrow \infty} f^{(m)}(t, x)=f(t, x)$, for all $(t, x) \in[0, T) \times(0, \infty)$.

Proof. See Appendix C.

\section{Proof of Theorem 5.1}

In this section, we use the results in Section 7 to finish the proof of Theorem 5.1 . 
Under the conditions of Theorem 5.1, from Proposition 4.2, we know the indirect absolute risk tolerance function

$$
g \in C^{1, \infty}([0, T) \times(0, \infty)) \cap C([0, T] \times(0, \infty)),
$$

and satisfies PDE

$$
\frac{1}{2}|\theta|^{2} g^{2} g_{x x}+r x g_{x}+g_{t}-r g=0
$$

on $[0, T) \times(0, \infty)$, with terminal condition

$$
g(T, x)=-\frac{V^{\prime}(x)}{V^{\prime \prime}(x)}, \quad \text { for all } x>0 .
$$

Since $V$ satisfies Assumption 5.1 and $U$ is more risk averse than $V$, we know $U$ satisfies Assumption 3.1. Let the approximating sequence $\left\{f^{(m)}, m \geq 2\right\}$ of $f$ be constructed as in Section 7 ,

Lemma 8.1 Under Assumptions 2.1] and 5.1, if $-\frac{U^{\prime}(x)}{U^{\prime \prime}(x)}<-\frac{V^{\prime}(x)}{V^{\prime \prime}(x)}$, for all $x>0$, then for each $m \geq 2, f^{(m)}(t, x) \leq g(t, x)$, on $\mathcal{D}^{(m)}$.

Proof. For each $m \geq 2$, set

$$
\begin{aligned}
\mathcal{B}_{0}^{(m)} & =\left\{\left(t, \frac{1}{m} e^{-\int_{t}^{T} r(s) d s}\right): t \in[0, T]\right\}, \\
\mathcal{B}_{1}^{(m)} & =\left\{\left(t, m e^{-\int_{t}^{T} r(s) d s}\right): t \in[0, T]\right\},
\end{aligned}
$$

then $\overline{\mathcal{D}^{(m)}}=\mathcal{D}^{(m)} \cup \partial^{*} \mathcal{D}^{(m)}$, where $\overline{\mathcal{D}^{(m)}}$ denotes the closure of $\mathcal{D}^{(m)}$ and $\partial^{*} \mathcal{D}^{(m)}=$ $\mathcal{B}_{0}^{(m)} \cup \mathcal{T}^{(m)} \cup \mathcal{B}_{1}^{(m)}$. The function $f^{(m)}$ is originally defined on $\mathcal{D}^{(m)} \cup \mathcal{T}^{(m)}$. Now we extend its definition to $\overline{\mathcal{D}^{(m)}}$ as follows: $f^{(m)}(t, x)=0$, for all $(t, x) \in \mathcal{B}_{0}^{(m)} \cup \mathcal{B}_{1}^{(m)}$ such that $t<T$, and

$$
f^{(m)}\left(T, \frac{1}{m}\right)=-\frac{U^{\prime}\left(\frac{1}{m}\right)}{U^{\prime \prime}\left(\frac{1}{m}\right)}, \quad f^{(m)}(T, m)=-\frac{U^{\prime}(m)}{U^{\prime \prime}(m)} .
$$

With this extension, we can see from Proposition 7.2 that $f^{(m)}$ is an upper semicontinuous function 5 on $\overline{\mathcal{D}^{(m)}}$.

Let $h=f^{(m)}-g$, then $h$ is upper semi-continuous on $\overline{\mathcal{D}^{(m)}}$. Since $-\frac{U^{\prime}(x)}{U^{\prime \prime}(x)}<$ $-\frac{V^{\prime}(x)}{V^{\prime \prime}(x)}$, for all $x>0$, we know $h<0$ on $\partial^{*} \mathcal{D}^{(m)}$. Obviously, $\partial^{*} \mathcal{D}^{(m)}$ is a compact set,

\footnotetext{
${ }^{5}$ See Appendix D,
} 
and therefore, from Proposition D.2, we can see on $\partial^{*} \mathcal{D}^{(m)}, h$ attains its maximum at some $\left(t_{0}, x_{0}\right) \in \partial^{*} \mathcal{D}^{(m)}$, which implies $h(t, x) \leq-\varepsilon<0$, for all $(t, x) \in \partial^{*} \mathcal{D}^{(m)}$, where $\varepsilon=-h\left(t_{0}, x_{0}\right)>0$. Moreover, there exists a constant $\delta>0$ such that 6 $h(t, x)<-\frac{\varepsilon}{2}<0$, for all $(t, x) \in \mathcal{O}^{(m)}(\delta) \cap \overline{\mathcal{D}^{(m)}}$, where

$$
\begin{aligned}
\mathcal{O}^{(m)}(\delta) & =\mathcal{O}_{1}^{(m)}(\delta) \cup \mathcal{O}_{2}^{(m)}(\delta) \cup \mathcal{O}_{3}^{(m)}(\delta), \\
\mathcal{O}_{1}^{(m)}(\delta) & =\left\{(t, x): t \in[0, T], \frac{1}{m} e^{-\int_{t}^{T} r(s) d s} \leq x<\frac{1}{m} e^{-\int_{t}^{T} r(s) d s}+\delta\right\}, \\
\mathcal{O}_{2}^{(m)}(\delta) & =(T-\delta, T] \times\left(\frac{1}{m}, m\right), \\
\mathcal{O}_{3}^{(m)}(\delta) & =\left\{(t, x): t \in[0, T], m e^{-\int_{t}^{T} r(s) d s}-\delta<x \leq m e^{-\int_{t}^{T} r(s) d s}\right\} .
\end{aligned}
$$

Obviously, $\mathcal{D}^{(m)} \backslash \mathcal{O}^{(m)}(\delta)$ is a compact set, then there exists a constant $\alpha$ such that

$$
-\alpha+\frac{1}{2}|\theta|^{2}\left(f^{(m)}+g\right) g_{x x}-r<0, \quad \text { on } \mathcal{D}^{(m)} \backslash \mathcal{O}^{(m)}(\delta) .
$$

Suppose, to the contrary, that $f^{(m)}>g$ somewhere in $\mathcal{D}^{(m)}$. Consider function $w$ defined by $w=h e^{\alpha t}$, then $w<0$ on $\mathcal{O}^{(m)}(\delta) \cap \overline{\mathcal{D}^{(m)}}$ and $w>0$ somewhere in $\mathcal{D}^{(m)}$. Obviously, $w$ is upper semi-continuous, and therefore, by Proposition D.2, $w$ attains its positive maximum at $\left(t_{1}, x_{1}\right) \in \mathcal{D}^{(m)} \backslash \mathcal{O}^{(m)}(\delta)$. So, at $\left(t_{1}, x_{1}\right), w>0, w_{x}=0$, $w_{x x} \leq 0$, and $w_{t}=h_{t} e^{\alpha t}+\alpha h e^{\alpha t} \leq 0$. Consequently, at $\left(t_{1}, x_{1}\right), h>0, h_{x}=0$, $h_{x x} \leq 0$, and $h_{t} \leq-\alpha h$. Thus, at $\left(t_{1}, x_{1}\right)$, we have from (7.7) and (8.1) that7

$$
\begin{aligned}
0= & \frac{1}{2}|\theta|^{2}\left[\left(f^{(m)}\right)^{2} f_{x x}^{(m)}-g^{2} g_{x x}\right]+r x\left(f_{x}^{(m)}-g_{x}\right) \\
& +\left(f_{t}^{(m)}-g_{t}\right)-r\left(f^{(m)}-g\right) \\
= & \frac{1}{2}|\theta|^{2}\left(f^{(m)}\right)^{2} h_{x x}+r x h_{x}+h_{t}+\left[\frac{1}{2}|\theta|^{2}\left(f^{(m)}+g\right) g_{x x}-r\right] h \\
\leq & {\left[-\alpha+\frac{1}{2}|\theta|^{2}\left(f^{(m)}+g\right) g_{x x}-r\right] h } \\
< & 0,
\end{aligned}
$$

which leads to a contradiction.

${ }^{6}$ Otherwise, for each $k \geq 1$, there exists a $\left(t_{k}, x_{k}\right) \in \mathcal{O}^{(m)}\left(\frac{1}{k}\right) \cap \overline{\mathcal{D}^{(m)}}$ such that $h\left(t_{k}, x_{k}\right) \geq-\frac{\varepsilon}{2}$. It is not difficult to see that there is a subsequence of $\left\{\left(t_{k}, x_{k}\right), k \geq 1\right\}$, which is still denoted by $\left\{\left(t_{k}, x_{k}\right), k \geq 1\right\}$, converging to some $(s, y) \in \partial^{*} \mathcal{D}^{(m)}$. From the upper semi-continuity of $h$, we have $h(s, y) \geq \lim \sup _{k \rightarrow \infty} h\left(t_{k}, x_{k}\right) \geq-\frac{\varepsilon}{2}$, which is impossible, since $-\varepsilon$ is the maximum of $h$ on $\partial^{*} \mathcal{D}^{(m)}$.

${ }^{7}$ I would like to thank Yongsheng Song for a fruitful discussion. 
Lemma 8.2 Under Assumptions 2.1] and 5.1, if $-\frac{U^{\prime}(x)}{U^{\prime \prime}(x)}<-\frac{V^{\prime}(x)}{V^{\prime \prime}(x)}$, for all $x>0$, then $f(t, x) \leq g(t, x)$, on $[0, T) \times(0, \infty)$.

Proof. Proposition 7.3 and Lemma 8.1 combined yield the assertion.

Now we prove Theorem 5.1 as follows.

Proof of Theorem 5.1. For any $\varepsilon>0$, let $U^{\varepsilon}:(0, \infty) \rightarrow \mathbb{R}$ be a function such that its derivative $\dot{U}^{\varepsilon}(x)=U^{\prime}(x) e^{-\varepsilon x}$, for all $x \in(0, \infty)$. Obviously, $U^{\varepsilon}$ is a utility function and

$$
-\frac{\dot{U}^{\varepsilon}(x)}{\ddot{U}^{\varepsilon}(x)}=\frac{U^{\prime}(x)}{-U^{\prime \prime}(x)+\varepsilon U^{\prime}(x)}, \quad \text { for all } x>0,
$$

which implies

$$
-\frac{\dot{U}^{\varepsilon}(x)}{\ddot{U^{\varepsilon}}(x)}<-\frac{U^{\prime}(x)}{U^{\prime \prime}(x)} \leq-\frac{V^{\prime}(x)}{V^{\prime \prime}(x)}, \text { for all } x>0 .
$$

Corresponding to utility function $U^{\varepsilon}$, the indirect absolute risk tolerance function is denoted by $f^{\varepsilon}(t, x)$. By Lemma 8.2, we have

$$
f^{\varepsilon}(t, x) \leq g(t, x), \quad \text { for all }(t, x) \in[0, T) \times(0, \infty) .
$$

In order to complete the proof, it suffices to show $\lim _{\varepsilon \downarrow \downarrow} f^{\varepsilon}(t, x)=f(t, x)$, for all $t \in[0, T)$ and $x>0$.

Actually, $\dot{U}^{\varepsilon}(x) \uparrow U^{\prime}(x)$ and $-\frac{\dot{U}^{\varepsilon}(x)}{\dot{U}^{\varepsilon}(x)} \uparrow-\frac{U^{\prime}(x)}{U^{\prime \prime}(x)}$ as $\varepsilon \downarrow 0$, for all $x>0$. Let $I^{\varepsilon}$ denote the inverse marginal utility function of $U^{\varepsilon}$, that is, $\dot{U}^{\varepsilon}\left(I^{\varepsilon}(y)\right)=y$ for all $y>0$, then $I^{\varepsilon}(y) \uparrow I(y)$ as $\varepsilon \downarrow 0$, for all $y>0$. Let $\mu^{\varepsilon}(t, y)=\mathbb{E}_{t}\left[I^{\varepsilon}\left(y \frac{H(T)}{H(t)}\right) \frac{H(T)}{H(t)}\right]$, then $\mu^{\varepsilon}(t, y) \uparrow \mu(t, y)$ as $\varepsilon \downarrow 0$, for all $(t, y) \in[0, T) \times(0, \infty)$. Let $\lambda^{\varepsilon}$ be defined by

$$
\mu^{\varepsilon}\left(t, \lambda^{\varepsilon}(t, x)\right)=x, \quad \text { for all }(t, x) \in[0, T) \times(0, \infty),
$$

then $\lambda^{\varepsilon}(t, x) \uparrow \lambda(t, x)$ as $\varepsilon \downarrow 0$, for all $(t, x) \in[0, T) \times(0, \infty)$. Consequently, we have

$$
-\frac{\dot{U}^{\varepsilon}\left(I^{\varepsilon}\left(\lambda^{\varepsilon}(t, x) \frac{H(T)}{H(t)}\right)\right)}{\ddot{U}^{\varepsilon}\left(I^{\varepsilon}\left(\lambda^{\varepsilon}(t, x) \frac{H(T)}{H(t)}\right)\right)} \frac{H(T)}{H(t)} \rightarrow-\frac{U^{\prime}\left(I\left(\lambda(t, x) \frac{H(T)}{H(t)}\right)\right)}{U^{\prime \prime}\left(I\left(\lambda(t, x) \frac{H(T)}{H(t)}\right)\right)} \frac{H(T)}{H(t)}
$$

almost surely as $\varepsilon \downarrow 0$, for all $(t, x) \in[0, T) \times(0, \infty)$. Moreover, by Proposition 4.1, we have

$$
f^{\varepsilon}(t, x)=\mathbb{E}_{t}\left[-\frac{\dot{U}^{\varepsilon}\left(I^{\varepsilon}\left(\lambda^{\varepsilon}(t, x) \frac{H(T)}{H(t)}\right)\right)}{\ddot{U}^{\varepsilon}\left(I^{\varepsilon}\left(\lambda^{\varepsilon}(t, x) \frac{H(T)}{H(t)}\right)\right)} \frac{H(T)}{H(t)}\right] .
$$

Then by the same way to prove Proposition 7.3, using the Dominated Convergence Theorem, we can have $\lim _{\varepsilon \downarrow 0} f^{\varepsilon}(t, x)=f(t, x)$, for all $t \in[0, T)$ and $x>0$. 


\section{Appendix}

\section{A Proof of Proposition 3.1}

Lemma A.1 If $U^{\prime}(0)=\infty$, then $\lim \inf _{x \downarrow 0}-\frac{U^{\prime}(x)}{U^{\prime \prime}(x)}=0$.

Proof. Suppose otherwise that $\liminf _{x \downarrow 0}-\frac{U^{\prime}(x)}{U^{\prime \prime}(x)}=A>0$, then there exists a $x_{0} \in(0,1)$ such that, $-\frac{U^{\prime}(x)}{U^{\prime \prime}(x)}>\frac{A}{2}$, for all $x \in\left(0, x_{0}\right)$. Then we have

$$
\left(\log U^{\prime}(x)\right)^{\prime}=\frac{U^{\prime \prime}(x)}{U^{\prime}(x)}>-\frac{2}{A}, \quad \text { for } x \in\left(0, x_{0}\right)
$$

and therefore,

$$
\log U^{\prime}(1)-\log U^{\prime}(x)=\int_{x}^{1} \frac{U^{\prime \prime}(z)}{U^{\prime}(z)} d z>\frac{2(x-1)}{A}, \quad \text { for } x \in\left(0, x_{0}\right)
$$

Thus $U^{\prime}(x)<U^{\prime}(1) e^{\frac{2(1-x)}{A}}$, for $x \in\left(0, x_{0}\right)$, which is impossible, since $U^{\prime}(0)=\infty$.

Lemma A.2 For any utility function $U$, there exist constants $c_{0}>0$ and $c_{1}>0$ such that

$$
|U(I(y))| \leq \max \left\{c_{0}+y, c_{0}+c_{1} I(y)\right\}, \quad \text { for all } y>0
$$

Proof. The concavity of $U$ implies

$$
U(I(y)) \leq U(1)+U^{\prime}(1)(I(y)-1), \quad \text { for all } y>0
$$

On the other hand, it is well know that $U(I(y))-y I(y)=\sup _{x>0}[U(x)-y x]$. Then

$$
-U(I(y)) \leq-y I(y)-U(1)+y \leq-U(1)+y, \quad \text { for all } y>0 .
$$

Finally, a combination of $(\underline{A .2})$ and $(\underline{A .3})$ yields the assertion.

Lemma A.3 Under Assumption 3.1, we have

$$
\begin{aligned}
U^{\prime}(x) & \leq U^{\prime}(1)\left(\frac{1+x}{2}\right)^{-\frac{1}{c}}, \quad \text { for all } x>1, \\
I(y) & \leq 2\left(U^{\prime}(1)\right)^{c} y^{-c}-1, \quad \text { for all } y \in\left(0, U^{\prime}(1)\right) .
\end{aligned}
$$


Proof. By Assumption 3.1,

$$
\left(\log U^{\prime}(x)\right)^{\prime}=\frac{U^{\prime \prime}(x)}{U^{\prime}(x)} \leq-\frac{1}{c(1+x)}, \quad \text { for all } x>0
$$

Then for all $x>1$,

$$
\log U^{\prime}(x)-\log U^{\prime}(1)=\int_{1}^{x}\left(\log U^{\prime}(z)\right)^{\prime} d z \leq-\int_{1}^{x} \frac{d z}{c(1+z)}=-\frac{1}{c} \log \frac{1+x}{2},
$$

yielding (A.4). Suppose $y<U^{\prime}(1)$, then $I(y)>1$, and therefore, by (A.4),

$$
y=U^{\prime}(I(y)) \leq U^{\prime}(1)\left(\frac{1+I(y)}{2}\right)^{-\frac{1}{c}},
$$

which implies (A.5).

Lemma A.4 Under Assumption 3.1, we have for all $a>0$ that

$$
\begin{aligned}
& \int_{-\infty}^{\infty} e^{z} I\left(e^{z}\right) e^{-a z^{2}} d z<\infty \\
& \int_{-\infty}^{\infty}\left|U\left(I\left(e^{z}\right)\right)\right| e^{-a z^{2}} d z<\infty .
\end{aligned}
$$

Proof. By Lemma A.3, we have

$$
I\left(e^{z}\right) \leq \begin{cases}2\left(U^{\prime}(1)\right)^{c} e^{-c z}-1, & \text { if } e^{z}<U^{\prime}(1) \\ 1, & \text { if } e^{z} \geq U^{\prime}(1),\end{cases}
$$

which obviously yields (A.6). A combination of (A.1) and (A.8) leads to (A.7).

We refer to Karatzas and Shreve [8, pp.254-255] for the following lemma:

Lemma A.5 Suppose $k: \mathbb{R} \rightarrow \mathbb{R}$ is a Borel-measurable function satisfying the condition

$$
\int_{-\infty}^{\infty}|k(z)| e^{-a z^{2}} d z<\infty
$$

for some $a>0$. Set

$$
\kappa(t, z)=\mathbb{E}[k(z+\sqrt{t} \xi)], \quad(t, z) \in\left[0, \frac{1}{2 a}\right) \times \mathbb{R}
$$

where $\xi \sim \mathcal{N}(0,1)$, the standard normal distribution. Then $\kappa$ has continuous derivatives of all orders, for all $t \in\left(0, \frac{1}{2 a}\right)$ and $z \in \mathbb{R}$. Moreover, if $f$ is continuous at $z_{0} \in \mathbb{R}$, then $\kappa$ is continuous at $\left(0, z_{0}\right)$. Particularly, if $f$ is continuous on $\mathbb{R}$, then $\kappa$ is continuous on $\left[0, \frac{1}{2 a}\right) \times \mathbb{R}$. 
Lemma A.6 Under Assumptions 2.1] and 3.1,

$$
\begin{aligned}
& \mu \in C^{1, \infty}([0, T) \times(0, \infty)) \cap C([0, T] \times(0, \infty)), \\
& \lambda \in C^{1, \infty}([0, T) \times(0, \infty)) \cap C([0, T] \times(0, \infty)) .
\end{aligned}
$$

Proof. Let $k(z)=e^{z} I\left(e^{z}\right)$, for all $z \in \mathbb{R}$. Obviously, $k(z)>0$, for all $z \in \mathbb{R}$. By Lemma A.4, $\int_{-\infty}^{\infty} k(z) e^{-a z^{2}} d z<\infty$, for all $a>0$. Set

$$
\kappa(t, z)=\mathbb{E}[k(z+\sqrt{t} \xi)], \quad(t, x) \in[0, \infty) \times \mathbb{R},
$$

where $\xi \sim \mathcal{N}(0,1)$. Then by Lemma A.5, $\kappa$ has continuous derivatives of all orders, for all $t \in(0, \infty)$ and $z \in \mathbb{R}$, and is continuous on $[0, \infty) \times \mathbb{R}$. Since

$$
\log \left(y \frac{H(T)}{H(t)}\right)=\log y-\int_{t}^{T} r(s) d s-\frac{1}{2} \Theta(t)-\int_{t}^{T} \theta(s)^{\top} d B(s),
$$

where $\Theta(t)=\int_{t}^{T}|\theta(s)|^{2} d s$, for all $t \in[0, T]$, we can see from (3.7) that

$$
\mu(t, y)=\frac{1}{y} \mathbb{E}_{t}\left[k\left(\log \left(y \frac{H(T)}{H(t)}\right)\right)\right]=\frac{1}{y} \kappa\left(\Theta(t), \log y-\int_{t}^{T} r(s) d s-\frac{1}{2} \Theta(t)\right),
$$

and therefore, (A.9) follows. Here we have used the fact that $\Theta$ is continuously differentiable on $[0, T]$, which is obvious under Assumption [2.1.

Now we prove $\lambda \in C^{1, \infty}([0, T) \times(0, \infty))$, using the Implicit Function Theorem 8 . To this end, we extent the definition of $\mu$ to a open domain containing $[0, T) \times(0, \infty)$. Let $\beta:(-\infty, T] \rightarrow[0, \infty)$ and $\gamma:(-\infty, T] \rightarrow \mathbb{R}$ be defined as follows:

$$
\begin{aligned}
& \beta(t)= \begin{cases}\Theta(t), & \text { for } t \in[0, T], \\
\Theta(0)-|\theta(0)|^{2} t, & \text { for } t<0,\end{cases} \\
& \gamma(t)= \begin{cases}\int_{t}^{T} r(s) d s, & \text { for } t \in[0, T], \\
\int_{0}^{T} r(s) d s-r(0) t, & \text { for } t<0 .\end{cases}
\end{aligned}
$$

Obviously, $\beta$ and $\gamma$ are continuously differentiable on $(-\infty, T]$. Let $\tilde{\mu}$ be defined by

$$
\tilde{\mu}(t, y)=\frac{1}{y} \kappa\left(\beta(t), \log y-\gamma(t)-\frac{1}{2} \beta(t)\right), \quad \text { for }(t, y) \in(-\infty, T] \times(0, \infty) .
$$

\footnotetext{
${ }^{8}$ See Zorich [14].
} 
Then $\tilde{\mu} \in C^{1, \infty}((-\infty, T) \times(0, \infty))$. It is not difficult to see that, for each $t \in$ $(-\infty, T]$,

$$
\tilde{\mu}(t, y)=\mathbb{E}\left[e^{-\gamma(t)-\frac{1}{2} \beta(t)+\sqrt{\beta(t)}} \xi I\left(y e^{-\gamma(t)-\frac{1}{2} \beta(t)+\sqrt{\beta(t)} \xi}\right)\right]
$$

is strictly decreasing with respect to $y$, on $(0, \infty)$. Let $\tilde{\lambda}$ be defined by

$$
\tilde{\mu}(t, \tilde{\lambda}(t, x))=x, \quad \text { for }(t, x) \in(-\infty, T] \times(0, \infty) .
$$

Then by the Implicit Function Theorem, $\tilde{\lambda} \in C^{1, \infty}((-\infty, T) \times(0, \infty))$. Moreover, it is easy to see that $\tilde{\mu}=\mu$, on $[0, T] \times(0, \infty)$, and therefore, recalling (3.8), $\tilde{\lambda}=\lambda$, on $[0, T] \times(0, \infty)$. Thus we have $\lambda \in C^{1, \infty}([0, T) \times(0, \infty))$.

It remains to show $\lambda$ is continuous at $(T, x)$, for all $x>0$. Let sequence $\left(t_{n}, x_{n}\right) \in$ $[0, T] \times(0, \infty)$ converge to $(T, x)$, for some $x>0$, we shall prove $\lim _{n \rightarrow \infty} \lambda\left(t_{n}, x_{n}\right)=$ $U^{\prime}(x)$. Suppose $\liminf _{n \rightarrow \infty} \lambda\left(t_{n}, x_{n}\right)<U^{\prime}(x)$, then there exists some $y_{0} \in\left(0, U^{\prime}(x)\right)$ such that $\liminf _{n \rightarrow \infty} \lambda\left(t_{n}, x_{n}\right)<y_{0}$, then

$$
x=\lim _{n \rightarrow \infty} \mu\left(t_{n}, \lambda\left(t_{n}, x_{n}\right)\right) \geq \liminf _{n \rightarrow \infty} \mu\left(t_{n}, y_{0}\right)=\mu\left(T, y_{0}\right)=I\left(y_{0}\right)>x,
$$

which leads to a contradiction. Thus $\liminf _{n \rightarrow \infty} \lambda\left(t_{n}, x_{n}\right)<U^{\prime}(x)$ is impossible. Similarly, we can prove $\lim \sup _{n \rightarrow \infty} \lambda\left(t_{n}, x_{n}\right)>U^{\prime}(x)$ is impossible. So we complete the proof.

Lemma A.7 Suppose Assumption 2.1 is satisfied and $q:(0, \infty) \rightarrow \mathbb{R}$ is a Borelmeasurable function satisfying the condition

$$
\int_{0}^{\infty}\left|q\left(e^{z}\right)\right| \exp \left\{-\frac{z^{2}}{2(\Theta(0)+\varepsilon)}\right\} d z<\infty, \text { for some } \varepsilon>0,
$$

where $\Theta(t)=\int_{t}^{T}|\theta(s)|^{2} d s$, for all $t \in[0, T]$. Let $\nu$ be defined by

$$
\nu(t, y)=\mathbb{E}_{t}\left[q\left(y \frac{H(T)}{H(t)}\right)\right], \quad(t, y) \in[0, T] \times(0, \infty) .
$$

Then $\nu \in C^{1, \infty}([0, T) \times(0, \infty))$. Moreover, if $q$ is continuous at $y_{0}>0$, then $\nu$ is continuous at $\left(T, y_{0}\right)$. Particularly, if $q$ is continuous on $(0, \infty)$, then $\nu \in$ $C([0, T] \times(0, \infty))$.

Proof. It is just similar to the proof of (A.9).

Based on the previous lemmas, we are able to prove Proposition 3.1 as follows. 
Proof of Proposition 3.1. Let $q(y)=U(I(y))$ and

$$
\nu(t, y)=\mathbb{E}_{t}\left[q\left(y \frac{H(T)}{H(t)}\right)\right]=\mathbb{E}_{t}\left[U\left(I\left(y \frac{H(T)}{H(t)}\right)\right)\right],
$$

then by Lemmas A.4 and A.7.

$$
\nu \in C^{1, \infty}([0, T) \times(0, \infty)) \cap C([0, T] \times(0, \infty)) .
$$

Moreover, recalling (3.9), $u(t, x)=\nu(t, \lambda(t, x))$. Then by Lemma A.6, we finish the proof.

\section{B Supplementary Data for Section 4}

Proof of Lemma 4.1. We can rewrite (4.1) as

$$
\hat{X}(T, x)=I\left(u_{x}(t, x) \frac{H(T)}{H(t)}\right) .
$$

Differentiating the preceding equality with respect to $x$ yields

$$
\frac{\partial \hat{X}(T, x)}{\partial x}=I^{\prime}\left(u_{x}(t, x) \frac{H(T)}{H(t)}\right) u_{x x}(t, x) \frac{H(T)}{H(t)} .
$$

Obviously, $\frac{\partial \hat{X}(T, x)}{\partial x}>0$ almost surely, for each $x>0$. Let function $l$ be defined by

$$
l(t, y)=\mathbb{E}_{t}\left[I^{\prime}\left(y \frac{H(T)}{H(t)}\right)\left(\frac{H(T)}{H(t)}\right)^{2}\right]
$$

for all $(t, y) \in[0, T] \times(0, \infty)$. We can see from Proposition [3.1, (B.1) and Lemma B.1 below that, for any $t \in[0, T]$,

$$
\mathbb{E}_{t}\left[\frac{\partial \hat{X}(T, x)}{\partial x} \frac{H(T)}{H(t)}\right]=u_{x x}(t, x) l\left(t, u_{x}(t, x)\right)
$$

is continuous with respect to $x$, on $(0, \infty)$. Moreover, for any $z>z_{0}>0$, we have 9

$$
\begin{aligned}
& \int_{z_{0}}^{z} \mathbb{E}_{t}\left[\frac{\partial \hat{X}(T, x)}{\partial x} \frac{H(T)}{H(t)}\right] d x \\
= & \mathbb{E}_{t}\left[\int_{z_{0}}^{z} \frac{\partial \hat{X}(T, x)}{\partial x} \frac{H(T)}{H(t)} d x\right] \quad \text { (by Fubini's Theorem) } \\
= & \mathbb{E}_{t}\left[\left(\hat{X}(T, z)-\hat{X}\left(T, z_{0}\right)\right) \frac{H(T)}{H(t)}\right] \\
= & z-z_{0}, \quad(\text { by (4.2) })
\end{aligned}
$$

\footnotetext{
${ }^{9}$ I would like to thank Jia-An Yan for a helpful suggestion.
} 
which leads to (4.3).

Lemma B.1 Under Assumptions 2.1 and 3.1, let function $l$ be defined by (B.2), then

$$
l \in C^{1, \infty}([0, T) \times(0, \infty)) \cap C([0, T] \times(0, \infty)) .
$$

Proof. Obviously, $I^{\prime}(y)<0$, for all $y>0$. For each $y>0$,

$$
-I^{\prime}(y)=-\frac{1}{U^{\prime \prime}(I(y))}=-\frac{U^{\prime}(I(y))}{y U^{\prime \prime}(I(y))} \leq c \frac{1+I(y)}{y},
$$

where the inequality follows from Assumption 3.1. Thus, for any $z \in \mathbb{R}$,

$$
0<-e^{2 z} I^{\prime}\left(e^{z}\right) \leq c\left(e^{z}+e^{z} I\left(e^{z}\right)\right)
$$

By Lemma A.4, $\int_{-\infty}^{\infty} q\left(e^{z}\right) e^{-a z^{2}} d z<\infty$, for all $a>0$, where $q(y)=-y^{2} I^{\prime}(y)$. Since

$$
l(t, y)=-\frac{\mathbb{E}_{t}\left[q\left(y \frac{H(T)}{H(t)}\right)\right]}{y^{2}}
$$

the proof can be finished by using Lemma A.7.

Proof of Proposition 4.2. By Lemma B.2 below, we only need to show $f \in$ $C([0, T] \times(0, \infty))$. Noting $I^{\prime}(y)=\frac{1}{U^{\prime \prime}(I(y))}$, we have from (4.6) that

$$
f(t, x)=\mathbb{E}_{t}\left[-\lambda(t, x) \frac{H(T)}{H(t)} I^{\prime}\left(\lambda(t, x) \frac{H(T)}{H(t)}\right) \frac{H(T)}{H(t)}\right],
$$

that is, $f(t, x)=-\lambda(t, x) l(t, \lambda(t, x))$, where $l$ is defined by (B.2). Lemmas B.1 and A.6 combined lead to $f \in C([0, T] \times(0, \infty))$.

Lemma B.2 Under Assumptions [2.1 and [3.1, $f \in C^{1, \infty}([0, T) \times(0, \infty))$, and satisfies PDE (4.7), on $[0, T) \times(0, \infty)$, with terminal condition (4.8).

Proof. By (3.11) and Proposition 3.1, it suffices to show $f$ satisfies PDE (4.7). By He and Huang [6, Proposition 2], $f$ satisfies the equation

$$
\frac{1}{2} \hat{\phi}^{\top} \sigma \sigma^{\top} \hat{\phi} f_{x x}+r x f_{x}+f_{t}-r f=0 .
$$

Substituting (3.12) into the previous equation yields (4.7). In the following, we give a direct proof. Substituting (3.12) into the HJB equation (3.10), we can have

$$
u_{t}+\left(r x+|\theta|^{2} f\right) u_{x}+\frac{1}{2}|\theta|^{2} f^{2} u_{x x}=0 .
$$


Noting $f^{2} u_{x x}=-f u_{x}=\frac{u_{x}^{2}}{u_{x x}}$, the preceding equation can be rewritten as

$$
u_{t}+r x u_{x}+\frac{1}{2}|\theta|^{2} f u_{x}=0
$$

Differentiating the previous equation with respect to $x$ yields

$$
u_{x t}+r u_{x}+r x u_{x x}+\frac{1}{2}|\theta|^{2} f_{x} u_{x}+\frac{1}{2}|\theta|^{2} f u_{x x}=0,
$$

and consequently,

$$
\frac{u_{x t}}{u_{x}}+\frac{1}{2}|\theta|^{2} f_{x}-\frac{r x}{f}+r-\frac{1}{2}|\theta|^{2}=0 .
$$

Differentiating the previous equation with respect to $x$ yields

$$
\frac{u_{x x t} u_{x}-u_{x t} u_{x x}}{u_{x}^{2}}+\frac{1}{2}|\theta|^{2} f_{x x}-\frac{r f-r x f_{x}}{f^{2}}=0 .
$$

Obviously,

$$
\frac{u_{x x t} u_{x}-u_{x t} u_{x x}}{u_{x}^{2}}=\frac{f_{t}}{f^{2}},
$$

which, together with (B.3), implies (4.7).

Lemma B.3 Under Assumptions 2.1 and 3.1, $\{f(s, \hat{X}(s)) H(s), s \in[t, T]\}$ is a local martingale, for each $t \in[0, T)$ and $x>0$.

Proof. The dynamics of $\{\hat{X}(s), s \in[t, T]\}$ is

$$
d \hat{X}(s)=\left[\hat{X}(s) r(s)+\hat{\phi}(s, \hat{X}(s))^{\top}(b(s)-r(s) \mathbb{1})\right] d s+\hat{\phi}(s, \hat{X}(s))^{\top} \sigma(s) d B(s)
$$

By the Itô's Formula,

$$
\begin{aligned}
& d f(s, \hat{X}(s)) \\
= & f_{s}(s, \hat{X}(s)) d s+f_{x}(s, \hat{X}(s))\left[\hat{X}(s) r(s)+\hat{\phi}(s, \hat{X}(s))^{\top}(b(s)-r(s) \mathbb{1})\right] d s \\
& +\frac{1}{2} f_{x x}(s, \hat{X}(s)) \hat{\phi}(s, \hat{X}(s))^{\top} \sigma(s) \sigma(s)^{\top} \hat{\phi}(s, \hat{X}(s)) d s \\
& +f_{x}(s, \hat{X}(s)) \hat{\phi}(s, \hat{X}(s))^{\top} \sigma(s) d B(s) .
\end{aligned}
$$

By the definition of $H(s)$, we have

$$
d H(s)=-r(s) H(s) d s-H(s) \theta(s)^{\top} d B(s)
$$


Therefore,

$$
\begin{aligned}
& d[f(s, \hat{X}(s)) H(s)] \\
= & f(s, \hat{X}(s)) d H(s)+H(s) d f(s, \hat{X}(s)) \\
& -f_{x}(s, \hat{X}(s)) \hat{\phi}(s, \hat{X}(s))^{\top} \sigma(s) \theta(s) H(s) d s \\
= & H(s)\left[\frac{1}{2} \hat{\phi}(s, \hat{X}(s))^{\top} \sigma(s) \sigma(s)^{\top} \hat{\phi}(s, \hat{X}(s)) f_{x x}(s, \hat{X}(s))\right. \\
& \left.+r(s) \hat{X}(s) f_{x}(s, \hat{X}(s))+f_{s}(s, \hat{X}(s))-r(s) f(s, \hat{X}(s))\right] d s \\
& + \text { a local martingale } \\
= & H(s)\left[\frac{1}{2}|\theta(s)|^{2} f^{2}(s, \hat{X}(s)) f_{x x}(s, \hat{X}(s))+r(s) \hat{X}(s) f_{x}(s, \hat{X}(s))\right. \\
& \left.+f_{s}(s, \hat{X}(s))-r(s) f(s, \hat{X}(s))\right] d s+\text { a local martingale, }
\end{aligned}
$$

where the last equality follows from (3.12). Then Lemma B.2 implies the $d s$-term is null and hence $\{f(s, \hat{X}(s)) H(s), s \in[t, T]\}$ is a local martingale.

\section{Supplementary Data for Section 7}

In this section, we use $\mathbf{1}_{A}$ to denote the indicator function of a set $A$.

\section{C.1 Proof of Proposition 7.1}

Proof of Proposition [7.1. Firstly, for each $m \geq 2$, the function $\frac{1}{m} \vee I(y) \wedge m$ is bounded and continuous. Then by similar discussions as in Lemma A.6, we can see

$$
\begin{aligned}
& \mu^{(m)} \in C^{1, \infty}([0, T) \times(0, \infty)) \cap C([0, T] \times(0, \infty)), \\
& \lambda^{(m)} \in C^{1, \infty}\left(\mathcal{D}^{(m)}\right) \cap C\left(\mathcal{D}^{(m)} \cup \mathcal{T}^{(m)}\right) .
\end{aligned}
$$

Finally, the assertion can be obtained by the same method to prove Proposition 3.1 .

\section{C.2 Proof of Proposition $\mathbf{7 . 2}$}

Lemma C.1 Suppose Assumptions 2.1 is satisfied. For each $m \geq 2$, let $\nu^{(m)}$ be defined by

$$
\nu^{(m)}(t, y)=\mathbb{E}_{t}\left[q^{(m)}\left(y \frac{H(T)}{H(t)}\right)\right], \quad \text { for all }(t, y) \in[0, T] \times(0, \infty),
$$


where

$$
q^{(m)}(y)=-\frac{U^{\prime}(I(y))}{U^{\prime \prime}(I(y))} y \mathbf{1}_{\left\{\frac{1}{m}<I(y)<m\right\}}, \quad \text { for all } y>0
$$

then $\nu^{(m)} \in C^{1, \infty}([0, T) \times(0, \infty))$ and $\nu^{(m)}$ is continuous at $(T, y)$, for all $y \in$ $(0, \infty) \backslash\left\{U^{\prime}(m), U^{\prime}\left(\frac{1}{m}\right)\right\}$.

Proof. Obviously, for each $m \geq 2, q^{(m)}$ is bounded on $(0, \infty)$ and is continuous on $(0, \infty) \backslash\left\{U^{\prime}(m), U^{\prime}\left(\frac{1}{m}\right)\right\}$. Then Lemma A.7 leads to the assertion.

Lemma C.2 Under Assumptions [2.1, for each $m \geq 2$,

$$
\begin{aligned}
& f^{(m)}(t, x) \\
= & \mathbb{E}_{t}\left[-\frac{U^{\prime}\left(I\left(\lambda^{(m)}(t, x) \frac{H(T)}{H(t)}\right)\right)}{U^{\prime \prime}\left(I\left(\lambda^{(m)}(t, x) \frac{H(T)}{H(t)}\right)\right)} \frac{H(T)}{H(t)} \mathbf{1}_{\left\{\frac{1}{m}<I\left(\lambda^{(m)}(t, x) \frac{H(T)}{H(t)}\right)<m\right\}}\right] \\
= & \mathbb{E}_{t}\left[-\frac{U^{\prime}\left(X^{(m)}(T)\right)}{U^{\prime \prime}\left(X^{(m)}(T)\right)} \frac{H(T)}{H(t)} \mathbf{1}_{\left\{\frac{1}{m}<X^{(m)}(T)<m\right\}}\right]
\end{aligned}
$$

for all $(t, x) \in \mathcal{D}^{(m)} \cup \mathcal{T}^{(m)}$.

Proof. In view of (7.3), we only need to prove (C.5). From the facts that $\lambda^{(m)}(t, x)=$ $u_{x}^{(m)}(t, x)$ and that

$$
\frac{U^{\prime}\left(I\left(\lambda^{(m)}(t, x) \frac{H(T)}{H(t)}\right)\right)}{U^{\prime \prime}\left(I\left(\lambda^{(m)}(t, x) \frac{H(T)}{H(t)}\right)\right)}=\lambda^{(m)}(t, x) \frac{H(T)}{H(t)} I^{\prime}\left(\lambda^{(m)}(t, x) \frac{H(T)}{H(t)}\right),
$$

we know it suffices to show, for each $m \geq 1$,

$$
u_{x x}^{(m)}(t, x) \mathbb{E}_{t}\left[\left(\frac{H(T)}{H(t)}\right)^{2} I^{\prime}\left(u_{x}^{(m)}(t, x) \frac{H(T)}{H(t)}\right) \mathbf{1}_{\left\{\frac{1}{m}<I\left(u_{x}^{(m)}(t, x) \frac{H(T)}{H(t)}\right)<m\right\}}\right]=1,
$$

for all $(t, x) \in \mathcal{D}^{(m)} \cup \mathcal{T}^{(m)}$.

Let $q^{(m)}$ and $\nu^{(m)}$ be defined by (C.4 $)$ and (C.3), respectively. Then

$$
q^{(m)}(y)=-y^{2} I^{\prime}(y) \mathbf{1}_{\left\{\frac{1}{m}<I(y)<m\right\}},
$$

and therefore,

$$
\nu^{(m)}(t, y)=-y^{2} \mathbb{E}_{t}\left[\left(\frac{H(T)}{H(t)}\right)^{2} I^{\prime}\left(y \frac{H(T)}{H(t)}\right) \mathbf{1}_{\left\{\frac{1}{m}<I\left(y \frac{H(T)}{H(t)}\right)<m\right\}}\right] .
$$


Let $\beta^{(m)}(t, x)$ denote the left-hand side of (C.7), then

$$
\beta^{(m)}(t, x)=-\frac{u_{x x}^{(m)}(t, x) \nu^{(m)}\left(t, u_{x}^{(m)}(t, x)\right)}{\left(u_{x}^{(m)}(t, x)\right)^{2}} .
$$

Proposition 7.1 and Lemma C.1 combined imply $\beta^{(m)} \in C\left(\mathcal{D}^{(m)} \cup \mathcal{T}^{(m)}\right)$.

On the other hand, for any given $t \in[0, T]$, and any $z_{0}$ and $z$ such that

$$
\frac{1}{m} e^{-\int_{t}^{T} r(s) d s}<z_{0}<z<m e^{-\int_{t}^{T} r(s) d s},
$$

we have

$$
\begin{aligned}
& \int_{z_{0}}^{z} \beta^{(m)}(t, x) d x \\
= & \mathbb{E}_{t}\left[\int_{z_{0}}^{z} u_{x x}^{(m)}(t, x)\left(\frac{H(T)}{H(t)}\right)^{2} I^{\prime}\left(u_{x}^{(m)}(t, x) \frac{H(T)}{H(t)}\right) \mathbf{1}_{\left\{\frac{1}{m}<I\left(u_{x}^{(m)}(t, x) \frac{H(T)}{H(t)}\right)<m\right\}} d x\right] \\
= & \mathbb{E}_{t}\left[\frac{H(T)}{H(t)} \int_{z_{0}}^{z} \mathbf{1}_{\left\{\frac{1}{m}<I\left(u_{x}^{(m)}(t, x) \frac{H(T)}{H(t)}\right)<m\right\}} d I\left(u_{x}^{(m)}(t, x) \frac{H(T)}{H(t)}\right)\right] \\
= & \mathbb{E}_{t}\left[\frac{H(T)}{H(t)}\left(\frac{1}{m} \vee I\left(u_{x}^{(m)}(t, z) \frac{H(T)}{H(t)}\right) \wedge m-\frac{1}{m} \vee I\left(u_{x}^{(m)}\left(t, z_{0}\right) \frac{H(T)}{H(t)}\right) \wedge m\right)\right] \\
= & z-z_{0},
\end{aligned}
$$

where the first equality follows from the Fubini's Theorem, and the last equality follows from (7.4) and the fact that $\lambda^{(m)}(t, x)=u_{x}^{(m)}(t, x)$. So, for any given $t \in$ $[0, T]$, we have $\beta^{(m)}(t, x)=1$, for all $x \in\left(\frac{1}{m} e^{-\int_{t}^{T} r(s) d s}, m e^{-\int_{t}^{T} r(s) d s}\right)$, which is desired.

Lemma C.3 Under Assumption 2.1, for each given $m \geq 2$ and $t \in[0, T)$,

$$
\begin{aligned}
\lim _{(s, x) \rightarrow\left(t, \frac{1}{m} e^{-J_{t}^{T} r(s) d s}\right)} \lambda^{(m)}(s, x) & =\infty, \\
\lim _{(s, x) \rightarrow\left(t, m e^{-\int_{t}^{T} r(s) d s}\right)} \lambda^{(m)}(s, x) & =0 .
\end{aligned}
$$

Proof. We only prove ([C.9), since the proof of $(\underline{\mathbb{C} .8})$ is similar. Suppose, to the contrary, that

$$
\limsup _{(s, x) \rightarrow\left(t, m e^{-\int_{t}^{T} r(s) d s}\right)} \lambda^{(m)}(s, x)>0,
$$


then there exist a constant $\varepsilon>0$ and a sequence $\left\{\left(s_{k}, x_{k}\right), k \geq 1\right\} \subset \mathcal{D}^{(m)} \cup \mathcal{T}^{(m)}$ converging to $\left(t, m e^{-\int_{t}^{T} r(s) d s}\right)$ such that $\lambda^{(m)}\left(s_{k}, x_{k}\right)>\varepsilon$, for all $k$. Thus, by relation (7.6),

$$
x_{k}=\mu^{(m)}\left(s_{k}, \lambda^{(m)}\left(s_{k}, x_{k}\right)\right)<\mu^{(m)}\left(s_{k}, \varepsilon\right) .
$$

By (C.1), letting $k \rightarrow \infty$ yields

$$
m e^{-\int_{t}^{T} r(s) d s} \leq \mu^{(m)}(t, \varepsilon)<m e^{-\int_{t}^{T} r(s) d s},
$$

which leads to a contradiction. So, (C.10) is impossible, and therefore, (C.9) is proved.

Lemma C.4 Under Assumption [2.1, for each given $m \geq 2$,

$$
\begin{aligned}
& \liminf _{(s, x) \rightarrow\left(T, \frac{1}{m}\right)} \lambda^{(m)}(s, x) \geq U^{\prime}\left(\frac{1}{m}\right), \\
& \limsup _{(s, x) \rightarrow(T, m)} \lambda^{(m)}(s, x) \leq U^{\prime}(m) .
\end{aligned}
$$

Proof. We only prove (C.12), since the proof of (C.11) is similar. Suppose, to the contrary, that

$$
\limsup _{(s, x) \rightarrow(T, m)} \lambda^{(m)}(s, x)>U^{\prime}(m)
$$

then there exist a constant $\varepsilon>0$ and a sequence $\left\{\left(s_{k}, x_{k}\right), k \geq 1\right\} \subset \mathcal{D}^{(m)} \cup \mathcal{T}^{(m)}$ converging to $(T, m)$ such that $U^{\prime}(m)+\varepsilon<U^{\prime}\left(\frac{1}{m}\right)$ and $\lambda^{(m)}\left(s_{k}, x_{k}\right)>U^{\prime}(m)+\varepsilon$, for all $k$. Thus, by relation (7.6),

$$
x_{k}=\mu^{(m)}\left(s_{k}, \lambda^{(m)}\left(s_{k}, x_{k}\right)\right)<\mu^{(m)}\left(s_{k}, U^{\prime}(m)+\varepsilon\right) .
$$

By (C.1), letting $k \rightarrow \infty$ yields

$$
m \leq \mu^{(m)}\left(T, U^{\prime}(m)+\varepsilon\right)=I\left(U^{\prime}(m)+\varepsilon\right)<m
$$

which leads to a contradiction. So, (C.13) is impossible, and therefore, (C.12) is proved.

Proof of Proposition 7.2. From Proposition 7.1, we can see $f^{(m)} \in C^{1, \infty}\left(\mathcal{D}^{(m)}\right)$. According to a similar way to prove Proposition 4.2, we can show $f^{(m)}$ satisfies PDE (17.7), on $\mathcal{D}^{(m)}$, with terminal condition (7.8). So, it suffices to show $f^{(m)}$ is 
continuous at $(T, x)$, for all $x \in\left(\frac{1}{m}, m\right)$, satisfies boundary conditions in (7.9), and satisfies (7.10) and (7.11).

Let $\nu^{(m)}$ be defined by (C.3), then by Lemma C.2.

$$
f^{(m)}(t, x)=\frac{\nu^{(m)}\left(t, \lambda^{(m)}(t, x)\right)}{\lambda^{(m)}(t, x)}, \quad \text { for all }(t, x) \in \mathcal{D}^{(m)} \cup \mathcal{T}^{(m)}
$$

From (C.2) and Lemma C.1, we can see $f^{(m)}$ is continuous at $(T, x)$, for all $x \in$ $\left(\frac{1}{m}, m\right)$.

In view of (C.5), we can obtain (7.9) from a combination of Lemma C.3 and the Dominated Convergence Theorem.

From Lemma C.4, we have

$$
\limsup _{(s, x) \rightarrow(T, m)} \lambda^{(m)}(s, x) \frac{H(T)}{H(t)} \leq U^{\prime}(m), \quad \text { almost surely, }
$$

and therefore,

$$
\liminf _{(s, x) \rightarrow(T, m)} I\left(\lambda^{(m)}(s, x) \frac{H(T)}{H(t)}\right) \geq m, \quad \text { almost surely. }
$$

Recalling (17.3), we can see the preceding inequality implies

$$
\lim _{(s, x) \rightarrow(T, m)} X^{(m)}(T)=m, \quad \text { almost surely. }
$$

Then from (․6 $)$ and the Fatou's Lemma, we can get

$$
\limsup _{(s, x) \rightarrow(T, m)} f^{(m)}(s, x) \leq-\frac{U^{\prime}(m)}{U^{\prime \prime}(m)}
$$

Moreover, (7.8) yields

$$
\lim _{x \uparrow m} f^{(m)}(T, x)=-\frac{U^{\prime}(m)}{U^{\prime \prime}(m)} .
$$

Thus (7.11) is obtained. The proof of (7.10) is similar.

\section{C.3 Proof of Proposition 7.3}

Lemma C.5 Under Assumptions [2.1 and 3.1, $\lim _{m \rightarrow \infty} \mu^{(m)}(t, y)=\mu(t, y)$, for any $(t, y) \in[0, T) \times(0, \infty)$. 
Proof. For any $m \geq 2$,

$$
\frac{H(T)}{H(t)}\left(\frac{1}{m} \vee I\left(y \frac{H(T)}{H(t)}\right) \wedge m\right) \leq \frac{H(T)}{H(t)}+\frac{H(T)}{H(t)} I\left(y \frac{H(T)}{H(t)}\right) .
$$

Then in view of (17.5) and (3.7), the assertion can be obtained from the Dominated Convergence Theorem.

Lemma C.6 Under Assumptions 2.1 and 3.1, $\lim _{m \rightarrow \infty} \lambda^{(m)}(t, x)=\lambda(t, x)$, for any $(t, x) \in \mathcal{D}^{(m)}$.

Proof. Given $(t, x) \in \mathcal{D}^{(m)}$, suppose, to the contrary, that either

$$
\liminf _{m \rightarrow \infty} \lambda^{(m)}(t, x)<\lambda(t, x)
$$

or

$$
\limsup _{m \rightarrow \infty} \lambda^{(m)}(t, x)>\lambda(t, x) .
$$

If $\liminf _{m \rightarrow \infty} \lambda^{(m)}(t, x)<\lambda(t, x)$, then there exist a constant $\varepsilon>0$ and a subsequence of $\left\{\lambda^{(m)}(t, x), m \geq 2\right\}$, which is still denoted by $\left\{\lambda^{(m)}(t, x), m \geq 2\right\}$, such that $\lambda(t, x)-\varepsilon>0$ and $\lambda^{(m)}(t, x)<\lambda(t, x)-\varepsilon$ for any $m$. Therefore, by relation (7.6),

$$
x=\mu^{(m)}\left(t, \lambda^{(m)}(t, x)\right)>\mu^{(m)}(t, \lambda(t, x)-\varepsilon) .
$$

By Lemma C.5, letting $m \rightarrow \infty$ yields $x \geq \mu(t, \lambda(t, x)-\varepsilon)>x$, which leads a contradiction. Thus $\lim \inf _{m \rightarrow \infty} \lambda^{(m)}(t, x)<\lambda(t, x)$ is impossible. By the same way, we can show $\limsup _{m \rightarrow \infty} \lambda^{(m)}(t, x)>\lambda(t, x)$ is also impossible.

Based on Lemma C.6, we can prove Proposition 7.3 as follows.

Proof of Proposition 17.3. First of all, for any given $(t, x) \in[0, T) \times(0, \infty)$, we can see from Lemma C.6 that

$$
\begin{array}{r}
-\frac{U^{\prime}\left(I\left(\lambda^{(m)}(t, x) \frac{H(T)}{H(t)}\right)\right)}{U^{\prime \prime}\left(I\left(\lambda^{(m)}(t, x) \frac{H(T)}{H(t)}\right)\right)} \frac{H(T)}{H(t)} \mathbf{1}_{\left\{\frac{1}{m}<I\left(\lambda^{(m)}(t, x) \frac{H(T)}{H(t)}\right)<m\right\}} \\
\rightarrow \quad-\frac{U^{\prime}\left(I\left(\lambda(t, x) \frac{H(T)}{H(t)}\right)\right)}{U^{\prime \prime}\left(I\left(\lambda(t, x) \frac{H(T)}{H(t)}\right)\right)} \frac{H(T)}{H(t)}, \quad \text { almost surely, }
\end{array}
$$


as $m \rightarrow \infty$. Then by Assumption 3.1,

$$
\begin{aligned}
& -\frac{U^{\prime}\left(I\left(\lambda^{(m)}(t, x) \frac{H(T)}{H(t)}\right)\right)}{U^{\prime \prime}\left(I\left(\lambda^{(m)}(t, x) \frac{H(T)}{H(t)}\right)\right)} \frac{H(T)}{H(t)} 1_{\left\{\frac{1}{m}<I\left(\lambda^{(m)}(t, x) \frac{H(T)}{H(t)}\right)<m\right\}} \\
\leq & c\left(1+I\left(\lambda^{(m)}(t, x) \frac{H(T)}{H(t)}\right)\right) \frac{H(T)}{H(t)} \\
\leq & c\left(1+I\left(y_{0} \frac{H(T)}{H(t)}\right)\right) \frac{H(T)}{H(t)}
\end{aligned}
$$

where $y_{0}=\inf _{m \geq 2} \lambda^{(m)}(t, x)>0$. Finally, (C.5) and the Dominated Convergence Theorem combined yield

$$
f^{(m)}(t, x) \rightarrow \mathbb{E}_{t}\left[-\frac{U^{\prime}\left(I\left(\lambda(t, x) \frac{H(T)}{H(t)}\right)\right)}{U^{\prime \prime}\left(I\left(\lambda(t, x) \frac{H(T)}{H(t)}\right)\right)} \frac{H(T)}{H(t)}\right]=f(t, x),
$$

as $m \rightarrow \infty$.

\section{Upper Semi-Continuous Functions}

Let $\mathbb{M}$ be a normed space, and $|\cdot|$ denote the norm. A function $f$ defined on $\mathbb{M}$ is said to be upper semi-continuous at $x_{0} \in \mathbb{M}$ if, given $\varepsilon>0$, there exists a $\delta>0$ such that $f(x)-f\left(x_{0}\right)<\varepsilon$, for all $x \in \mathbb{M}$ such that $\left|x-x_{0}\right|<\delta$. A function $f$ is said to be upper semi-continuous on $\mathbb{M}$ if it is upper semi-continuous at every point of $\mathbb{M}$.

We refer to Luenberger [9, p.40] for the following two propositions:

Proposition D.1 A function $f$ defined on $\mathbb{M}$ is upper semi-continuous at $x_{0}$ if and only if $\lim \sup _{x \rightarrow x_{0}} f(x) \leq f\left(x_{0}\right)$.

Proposition D.2 An upper semi-continuous function achieves its maximum on any compact subset. 


\section{References}

[1] K. Arrow, Liquidity preference, in: Lecture VI in Lecture Notes for Economics 285, The Economics of Uncertainty, Stanford University, 1963, pp.33-53.

[2] C. Borell, Monotonicity properties of optimal investment strategies for log-Brownian asset prices, Math. Finance 17 (2007), 143-153.

[3] J. Cox, C. Huang, Optimal consumption and portfolio policies when asset prices follow a diffusion process, J. Econ. Theory 49 (1989), 33-83.

[4] J. Cox, H. Leland, Notes on dynamic investment strategies, in: Proceedings of Seminar on the Analysis of Securities Prices, Center for Research in Security Prices (CRSP), Graduate School of Business, University of Chicago, 1982.

[5] C. Gollier, The Economics of Risk and Time, The MIT Press, Cambridge, Massachusetts, 2001.

[6] H. He, C. Huang, Consumption-portfolio policies: an inverse optimal problem, J. Econ. Theory 62 (1994), 257-293.

[7] I. Karatzas, J. Lehoczky, S. Shreve, Optimal portfolio and consumption decisions for a small investor on a finite horizon, SIAM J. Control Optim. 25 (1987), 1557-1586.

[8] I. Karatzas, S. Shreve, Brownian Motion and Stochastic Calculus, 2nd edition, Springer-Verlag, Berlin, 1991.

[9] D. Luenberger, Optimization by Vector Space Methods, John Wileys \& Sons, New York, 1969.

[10] R. Merton, Lifetime portfolio selection under uncertainty: the continuous-time case, Rev. Econ. Statist. 51 (1969), 247-257.

[11] R. Merton, Optimum consumption and portfolio rules in a continuous-time model, J. Econ. Theory 3 (1971), 373-413.

[12] S. Pliska, A stochastic calculus model of continuous trading: optimal portfolio, Math. Oper. Res. 11 (1986), 371-382.

[13] J. Pratt, Risk aversion in the small and in the large, Econometrica 32 (1964), 122-136.

[14] V. Zorich, Mathematical Analysis I, Springer-Verlag, Berlin, 2004. 